\title{
Quantum Antiferromagnetism in Quasicrystals
}

\author{
Stefan Wessel ${ }^{1}$, Anuradha Jagannathan ${ }^{2}$, and Stephan Haas ${ }^{2}$ \\ ${ }^{1}$ Institut für Theoretische Physik, ETH-Hönggerberg, CH-8093 Zürich, Switzerland \\ ${ }^{2}$ Department of Physics and Astronomy, University of Southern California, Los Angeles, CA 90089-0484
}

(November 20, 2018)

\begin{abstract}
The antiferromagnetic Heisenberg model is studied on a two-dimensional bipartite quasiperiodic lattice. The distribution of local staggered magnetic moments is determined on finite square approximants with up to 1393 sites, using the Stochastic Series Expansion Quantum Monte Carlo method. A non-trivial inhomogeneous ground state is found. For a given local coordination number, the values of the magnetic moments are spread out, reflecting the fact that no two sites in a quasicrystal are identical. A hierarchical structure in the values of the moments is observed which arises from the self-similarity of the quasiperiodic lattice. Furthermore, the computed spin structure factor shows antiferromagnetic modulations that can be measured in neutron scattering and nuclear magnetic resonance experiments. This generic model is a first step towards understanding magnetic quasicrystals such as the recently discovered $\mathrm{Zn}-\mathrm{Mg}-\mathrm{Ho}$ icosahedral structure.
\end{abstract}

PACS numbers:

Quantum magnetic phases of low-dimensional antiferromagnetic $(\mathrm{AF})$ Heisenberg systems are known to show various degrees of disorder caused by zero-point fluctuations. For example, isolated spin- $1 / 2$ chains have quasilong-ranged antiferromagnetic order [1], whereas two-leg ladders are short-range-ordered [2], and true long-range order is found in the two-dimensional square lattice [3]. While these states are homogeneous due to the translational invariance of the underlying lattice, it is interesting to explore how non-periodic environments, such as provided in quasicrystal structures, affect the magnetic properties of quantum magnets. Naturally, it can be expected that instead of a uniform order parameter, such as the staggered magnetization in a Heisenberg antiferromagnet, there will be instead a distribution of local order parameters. The magnitude of each local moment may, for example, strongly depend on its environment. In a local viewpoint, the magnetic moments on sites with a greater number of neighbors are expected to be suppressed due to increased local spin fluctuations. [4 This picture will be modified by the quasicrystalline equivalent of non-local spin-wave excitations, leading to a non-trivial distribution of the order parameters. Recent inelastic neutron scattering experiments on the $\mathrm{Zn}-\mathrm{Mg}$ Ho isocahedral quasicrystal [5] have revealed an antiferromagnetic superstructure, which fits very well with the antiferromagnetic Heisenberg model. As opposed to magnetic quasicrystals with itinerant charge carriers $\|6\|$, the electrons appear well localized in this material, and display an antiferromagnetic modulation with a large wave vector at temperatures below $6 \mathrm{~K}$, similar to the pattern of the generic quasicrystal structure reported here.

In this work we use the recently developed Stochastic Series Expansion Quantum Monte Carlo (SSE-QMC) algorithm [7] to analyze the magnetic ground state properties of the nearest-neighbor AF spin- $1 / 2$ Heisenberg model,

$$
H=J \sum_{\langle i, j\rangle} \mathbf{S}_{i} \cdot \mathbf{S}_{j}
$$

on a two-dimensional quasiperiodic tiling. This bipartite structure, shown in Fig. 1, is called "octagonal tiling" due to its overall 8-fold symmetry. Sites in this tiling have coordination numbers $z$ ranging from 3 to 8 . This generic lattice structure was chosen to ensure that the magnetic ground state is unfrustrated due to the bipartite property, i.e. nearest-neighbor sites belong to two distinct subtilings. [8] In the numerical study, approximants with $N=41,239$, and 1393 sites [9] are considered, and toroidal boundary conditions are applied. [10] The temperature is chosen low enough to obtain the ground state properties of these finite systems.

(a)

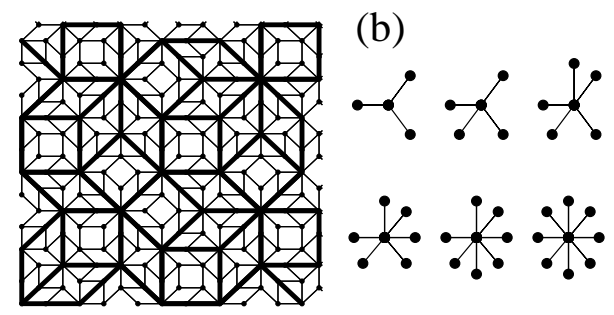

FIG. 1. Approximants of the octagonal tiling. (a) Inflation from 239 (thin lines) to 41 (thick lines) sites. (b) "Heisenberg stars" are the local building blocks.

An important property of quasicrystals, in the absence of invariance under translations, is their self-similarity under inflation transformations. These are site decimation operations that increase the length scale but globally preserve the quasiperiodic structure. An example of inflation is shown in Fig. 1(a) where original tiles and inflated tiles are superimposed. Since periodic approximants are finite pieces of the octagonal tiling, they are 
not invariant under such a transformation, but instead transform into smaller approximants. In this process, the sites which remain after an inflation operation can change their connectivity. A site of coordination number $\mathrm{z}$ will be transformed into a site of new coordination number $z^{\prime}$ after inflation, with $z^{\prime} \leq z$. We are interested in how this self-similarity is reflected in the inhomogeneous magnetic ground state of the Heisenberg Hamiltonian.

In the SSE-QMC simulations the local value of the staggered magnetic moment is determined at each lattice site $i$. It is defined by

$$
m(i)=\sqrt{\frac{3}{N} \sum_{j=1}^{N}(-1)^{i+j}\left\langle S_{i}^{z} S_{j}^{z}\right\rangle},
$$

where the sum extends over all lattice sites $\mathrm{j}$ of the approximant. The inset of Fig. 2 shows a finite-size extrapolation of the spatially averaged staggered magnetic moment, $\bar{m}=\sqrt{\left(3 / N^{2}\right) \sum(-1)^{i+j}\left\langle S_{i}^{z} S_{j}^{z}\right\rangle}$, where the sum extends over all pairs of sites $i$ and $j$. In the thermodynamic limit, this observable approaches $\bar{m}=$ $0.337 \pm 0.002$, indicating that the system has AF longrange order. Note that the average moment is larger than that of the square lattice, $\bar{m}>m_{s}=0.3071 \pm 0.0003$ [1], even though the average connectivity for the octagonal tiling is exactly $\bar{z}=4$, same as for the square lattice. This suggests that quantum fluctuations reducing the order parameter are suppressed due to the inhomogeneous connectivity in the octagonal tiling.

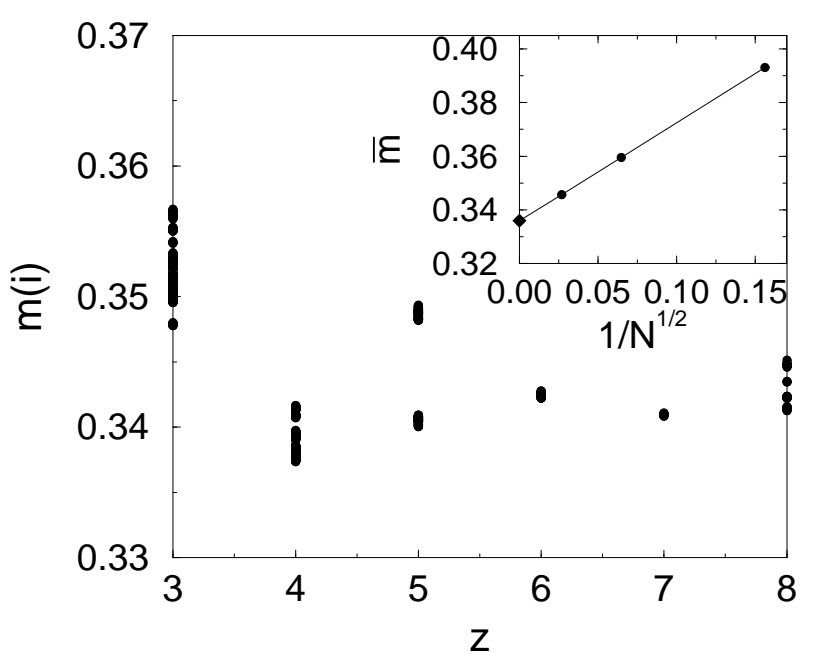

FIG. 2. Dependence of the staggered magnetic moments on the coordination number $z$. Inset: finite-size extrapolation of the average staggered magnetic moment.

The measured dependence of $m(i)$ on the local coordination number $z$ is plotted in the main part of Fig. 2 for the $\mathrm{N}=1393$ approximant. A wide spread of these moments is observed, particularly for small values of $z$. There is a tendency for sites with more neighbors to have smaller staggered moments because their spin is suppressed by forming a larger number of local bonds. [4] However, there are exceptions to this rule, indicating the presence of longer-range correlations. A further striking feature observed in Fig. 2 are splittings in the distribution function for the staggered moments. This is most evident at $z=5$, but it also occurs on smaller scales at other coordination numbers, e.g. at $z=8$.

Local environments clearly play an important part. To sort out the roles of the local versus the long-range correlations, we next focus on the local approximation to the estimator for the staggered moment,

$$
m_{\text {loc }}(i)=\sqrt{\frac{3}{z+1}\left(\frac{1}{4}-\sum_{j=1}^{z}\left\langle S_{i}^{z} S_{j}^{z}\right\rangle\right)},
$$

where the sum is truncated to the sites in the immediate vicinity of $i$. This quantity is a local approximation of $m(i)$ defined in Eq. (2). It reflects the average bond strength $(1 / z) \sum_{j=1}^{z} \mathbf{S}_{i} \cdot \mathbf{S}_{j}$ of site $i$ with its $z$ neighbors.

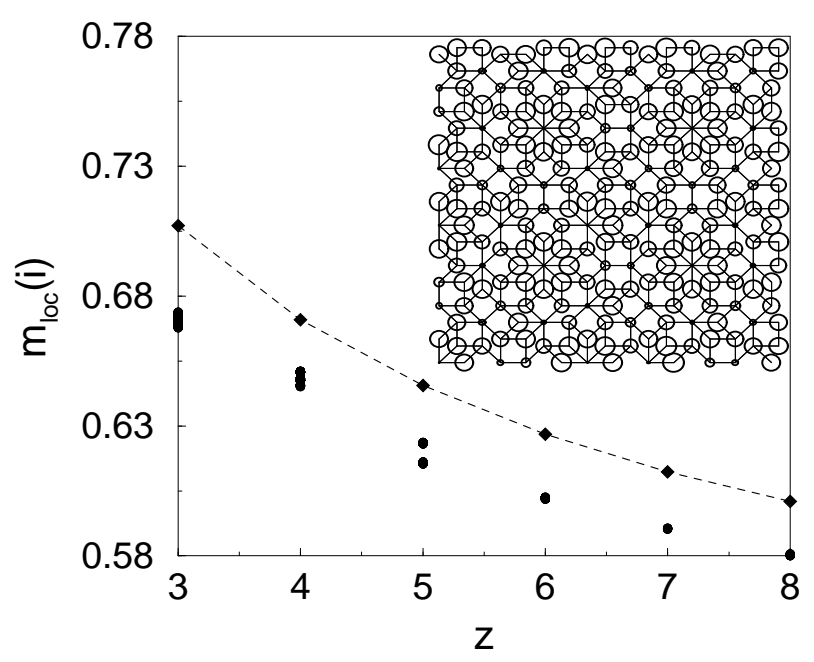

FIG. 3. Dependence of the local staggered magnetic moments on the coordination number $z$. The points on the dashed line are exact values obtained for Heisenberg stars. Inset: spatial variation of the local approximation to the local staggered moments where the radii of the circles correspond to the size of local staggered moments.

Fig. 3 shows a clear monotonic decay in $m_{l o c}(i)$ with increasing number of neighbors $z$. This effect can be understood by considering the local constituents of the cluster, shown in Fig. 1(b), which we call Heisenberg stars (HS). These are described by the nearest-neighbor Hamiltonian $H^{H S}(z)=J \sum_{j=1}^{z} \mathbf{S}_{0} \cdot \mathbf{S}_{j}$, where the index 0 denotes the central spin of the cluster and the AF exchange integral is set to $J=1$. The ground state energy of these stars can be calculated exactly, and is given by $E_{0}^{H S}(z)=-(2+z) / 4$. The estimator for the local magnetization can be obtained directly from Eq. (3), 


$$
m_{\text {loc }}^{H S}(z)=\sqrt{\frac{1}{z+1}\left(\frac{3}{4}-E_{0}\right)}=\sqrt{\frac{5+z}{4(z+1)}},
$$

implying a $z^{-1 / 2}$-like decay, consistent with the trend of the QMC data observed in Fig. 3. The ground state energy as well as the magnetic moment of the isolated Heisenberg clusters are naturally larger than the equivalent quantities in the quasicrystal where the presence of additional bonds lowers the energy per site and further screens the local magnetization.

For all coordination numbers, there is some degree of spread in the distribution of $m_{l o c}(z)$. This phenomenon can be understood by considering non-local corrections to the magnetic moments of the isolated Heisenberg stars. Next-nearest-neighbor corrections to the local moments for the six types of sites $(z=3, \ldots, 8)$ can be obtained in a self-consistent manner by re-diagonalizing the Heisenberg stars, using local spin operators that are renormalized according to their specific environments, i.e. by replacing $\left\langle S_{i}^{z}\right\rangle$ by $m_{\text {loc }}^{H S}(z)$ with the appropriate coordination number to determine the renormalized matrix elements. This procedure naturally leads to larger spreads for sites with small $z$, since in the octagonal tiling they have a wider variety of local environments than the sites with large $z$, which is observed in Fig. 3.

Furthermore, one notices a prominent bimodal splitting effect at sites with $z=5$. In the octagonal tiling, these sites occur in pairs and have two distinct local environments. 12. The first type of $\mathrm{z}=5$ clusters has two $\mathrm{z}=4$ neighbors, two $\mathrm{z}=3$ neighbors, and one $\mathrm{z}=5$ neighbor. The other type has four $z=4$ neighbors and one $\mathrm{z}=5$ neighbor. Analogous to the discussion in the above paragraph, the next-nearest-neighbor correction to the staggered magnetic moment for the two types can be determined by diagonalization of the corresponding $6 \times 6$ matrices in the $S_{t o t}^{z}=(N-2) / 2$ subspace of these two $\mathrm{N}=6$-site Heisenberg stars, using the magnetization values $m_{\text {loc }}^{H S}(z)$ of the Heisenberg stars in Eq. 4. 13] Following this procedure, one finds two renormalized staggered moments with a splitting $\Delta m_{l o c}(5)=0.007$, which is in very good agreement with the numerically observed splitting for the $z=5$ sites in Fig. 3 .

Let us now turn to the hierarchical structure of the magnetic ground state that can be observed in the splittings of the staggered moments of the $z=8$ sites. These sites have eight-fold symmetry out to their third neighbor shell. Since their local environments are identical up to a larger distance compared to other sites, the spread in values of $m_{l o c}(i)$ is relatively small (Fig. 3). One can however divide these sites into four groups according to their transformation under inflation, i.e. their new local coordination numbers $z^{\prime}$. The $m(i)$ values consequently fall into four discrete groups $\left(z^{\prime}=5,6,7\right.$, and 8), as shown in Fig. 4. This hierarchical fine structure is analogous to the discussion of the $z=5$ sites, but occurs on a smaller scale. Interestingly, the most symmetric group with $z^{\prime}=8$ experiences an additional hyperfine splitting due to a four-fold subgrouping with $z^{\prime \prime}=5,6,7$, and 8 under further inflation. In the limit of infinite size, this leads to a multifractal distribution of $m(i)$ for this class of sites.

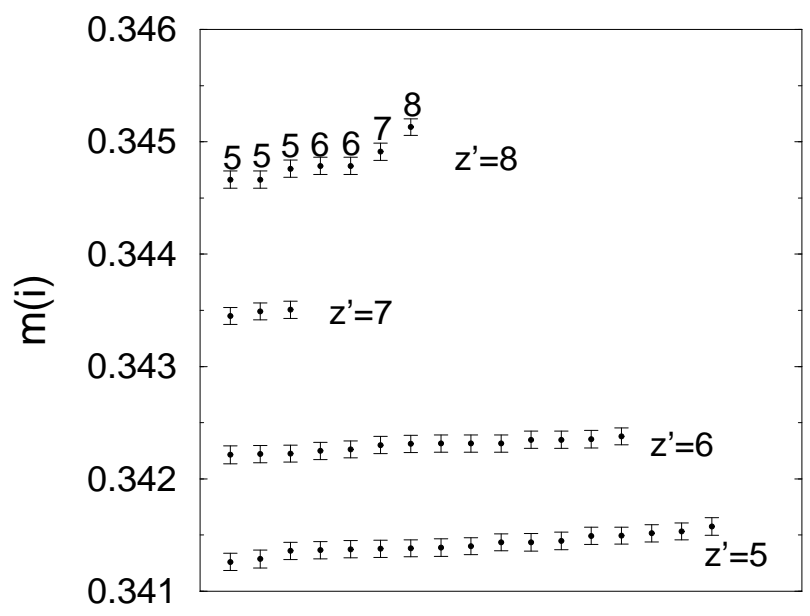

FIG. 4. Hierarchy of the staggered moment of the $z=8$ sites in the 1393 site tiling, grouped according to the value of $z^{\prime}$ under the inflation transformation. Numbers on top of the symbols give the value of $z^{\prime \prime}$ for the $z^{\prime}=8$ sites under a further inflation transformation.

To summarize this discussion, the different local environments in the quasiperiodic structures thus lead, firstly, to a systematic decrease of $m_{l o c}(i)$ with increasing $z$. The differences in next-nearest neighbor shells for a given $z$ gives rise to a spread of $m_{l o c}(i)$ and to discrete distributions which are self-similar on smaller and smaller scales, as seen in the 8-fold site example. These generic features should be observable by high-precision nuclear magnetic resonance measurements. Comparing the data in Figs. 2 and 3 one further observes that long-range correlations tend to suppress the differences in $m_{l o c}(i)$ between the sites, leading to a non-monotonous dependence of $m(i)$ on $z$. The spread in values of $m(i)$ is overall smaller than for $m_{l o c}(i)$. However, the splittings at $z=5$ and $z=8$ persist in $m(i)$, and should thus be experimentally observable.

We conclude the discussion of the magnetic ground state on the octagonal tiling by presenting the magnetic structure factor, which is of relevance to inelastic neutron scattering experiments. It is obtained by a Fourier transformation of the real space correlation function of the largest available approximant,

$$
S^{z z}(\mathbf{k})=\frac{1}{N} \sum_{\mathbf{r}, \mathbf{r}^{\prime}} e^{i \mathbf{k} \cdot\left(\mathbf{r}-\mathbf{r}^{\prime}\right)}\left\langle S_{\mathbf{r}}^{z} S_{\mathbf{r}^{\prime}}^{z}\right\rangle,
$$

using the QMC data for the octagonal tiling. In Fig. 5 the diffraction pattern of this quasicrystal lattice is compared with the antiferromagnetically induced superstructure. The eight-fold pattern in Fig. 5(a) reflects the 
eight-fold symmetry of the quasicrystal. The introduction of AF correlations leads to a splitting of Bragg peaks into the largest available wave vectors at the Brillouin zone boundary and their symmetry points, as it is seen in Fig. 5(b). For example, the central Bragg peak splits into $\mathbf{Q} \approx( \pm \pi, \pm \pi / 2)$ and $( \pm \pi / 2, \pm \pi)$, consistent with the modulation expected for the octagonal tiling. This $\mathrm{AF}$ superstructure is an extension to the two-dimensional case of the AF 1D structure discussed in [14].
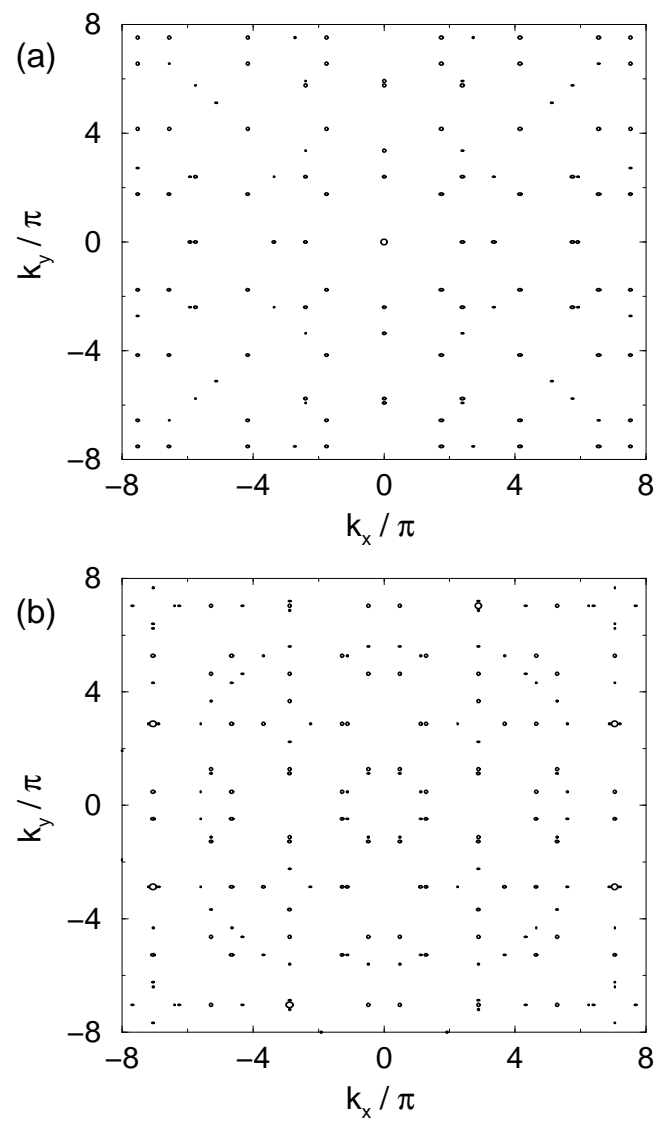

FIG. 5. (a) Simple Bragg scattering, and (b) antiferromagnetic superstructure in the octagonal tiling, extracted from Quantum Monte Carlo data on the 1393 site approximant.

In conclusion, we have examined the AF spin- $1 / 2$ Heisenberg model on the bipartite octagonal tiling, using the Stochastic Series Expansion Quantum Monte Carlo method. The main effects we observe are (i) an inhomogeneous distribution of staggered moments, (ii) a reduction of the local order parameter with increasing number of neighbors, (iii) strong long-range effects, (iv) evidence for hierarchical nature of the magnetic ground state, and (v) a magnetic superstructure in the spin structure factor. These predictions should be tested by highresolution NMR and neutron scattering measurements.

We thank A. Läuchli, O. Nohadani, B. Normand, R. Roscilde, and M. Sigrist for useful discussions, and ac- knowledge financial support by the Swiss National Fund, the Petroleum Research Fund, Grant No. ACS-PRF 35972-G6, and the Department of Energy, Grant No. DE-FG03-01ER45908. AJ acknowledges the computational facilities provided by IDRIS (Orsay). The numerical simulations were performed on the Asgard Beowulf cluster at the ETH Zürich and the hpc cluster at USC.

[1] J. des Cloiseaux and J. J. Pearson, Phys. Rev. 128, 2131 (1962).

[2] F. D. M. Haldane, Phys. Rev. Lett. 60, 1153 (1983); Phys. Lett. 93A, 464 (1983).

[3] E. Manousakis, Rev. Mod. Rhys. 63, 1 (1991), and references therein.

[4] N. Bulut, D. Hone, D. J. Scalapino, and E. Y. Loh, Phys. Rev. Lett. 62, 2192 (1989).

[5] T. J. Sato, H. Takakura, A. P. Tsai, K. Shibata, K. Ohoyama, and K. H. Andersen, Phys. Rev. B 61, 476 (2000).

[6] G. Trambly de Laissardière and D. Mayou, Phys. Rev. Lett. 85, 3273 (2000); M. A. Chernikov, A. Bernasconi, C. Beeli, A. Schilling, and H. R. Ott, Phys. Rev. B 48, 3058 (1993).

[7] A. W. Sandvik and J. Kurkijärvi, Phys. Rev. B 43, 5950 (1991); A. Sandvik, Phys. Rev. B 59 R14157 (1999).

[8] The present discussion focuses on unfrustrated lattices. Magnetic frustrations tend to introduce further disorder in the ground state, and may even destroy long-range magnetic order. Moreover, they introduce severe minus-sign problems to the Monte Carlo algorithm.

[9] M. Duneau, R. Mosseri, and C. Oguey, J. Phys. A 22, 4549 (1989).

[10] A. Jagannathan and H. J. Schulz, Phys. Rev. B 55, 8048 (1997).

[11] A. W. Sandvik, Phys. Rev. B 56, 11678 (1997).

[12] The members of a pair are inequivalent under inflation. One site is decimated, while the other remains as a 3 -fold site in the inflated tiling.

[13] In this calculation the moments of the isolated Heisenberg stars (Eq. 4) are used to determine the matrix elements. For example, the diagonal entries for the cluster with four $\mathrm{z}=4$ neighbors and one $\mathrm{z}=5$ neighbor are $H_{i i}=m_{\text {loc }}^{H S}(5)\left[ \pm m_{\text {loc }}^{H S}(5) \pm 4 m_{\text {loc }}^{H S}(4)\right]$.

[14] Ron Lifshitz, Material Science and Engineering A 294, 508 (2000). 